\title{
Characteristics of undiagnosed diseases network applicants: implications for referring providers
}

Nicole M. Walley', Loren D. M. Pena', Stephen R. Hooper², Heidi Cope'1, Yong-Hui Jiang ${ }^{1}$, Allyn McConkie-Rosell', Camilla Sanders ${ }^{1}$, Kelly Schoch ${ }^{1}$, Rebecca C. Spillmann ${ }^{1}$, Kimberly Strong ${ }^{3}$, Alexa T. McCray ${ }^{4}$, Paul Mazur ${ }^{4}$, Cecilia Esteves ${ }^{4}$, Kimberly LeBlanc ${ }^{4}$, Undiagnosed Diseases Network ${ }^{5}$, Anastasia L. Wise ${ }^{5}$ and Vandana Shashi ${ }^{{ }^{*}}$

\begin{abstract}
Background: The majority of undiagnosed diseases manifest with objective findings that warrant further investigation. The Undiagnosed Diseases Network (UDN) receives applications from patients whose symptoms and signs have been intractable to diagnosis; however, many UDN applicants are affected primarily by subjective symptoms such as pain and fatigue. We sought to characterize presenting symptoms, referral sources, and demographic factors of applicants to the UDN to identify factors that may determine application outcome and potentially differentiate between those with undiagnosed diseases (with more objective findings) and those who are less likely to have an undiagnosed disease (more subjective symptoms).
\end{abstract}

Methods: We used a systematic retrospective review of 151 consecutive Not Accepted and 50 randomly selected Accepted UDN applications. The primary outcome was whether an applicant was Accepted, or Not Accepted, and, if accepted, whether or not a diagnosis was made. Objective and subjective symptoms and information on prior specialty consultations were collected from provider referral letters. Demographic data and decision data on network acceptance were gathered from the UDN online portal.

Results: Fewer objective findings and more subjective symptoms were found in the Not Accepted applications. Not Accepted referrals also were from older individuals, reported a shorter period of illness, and were referred to the UDN by their primary care physicians. All of these differences reached statistical significance in comparison with Accepted applications. The frequency of subspecialty consults for diagnostic purposes prior to UDN application was similar in both groups.

Conclusions: The preponderance of subjective and lack of objective findings in the Not Accepted applications distinguish these from applicants that are accepted for evaluation and diagnostic efforts through the UDN. Not Accepted applicants are referred primarily by their primary care providers after multiple specialist consultations fail to yield answers. Distinguishing between patients with undiagnosed diseases with objective findings and those with primarily subjective findings can delineate patients who would benefit from further diagnostic processes from those who may have functional disorders and need alternative pathways for management of their symptoms.

Trial registration: clinicaltrials.gov NCT02450851, posted May 21st 2015.

Keywords: Medically unexplained symptoms, Medically unexplained physical symptoms, Undiagnosed diseases, Genomics, Health policy

\footnotetext{
* Correspondence: vandana.shashi@duke.edu

Deceased

${ }^{1}$ Division of Medical Genetics, Department of Pediatrics, Duke Health, Box

103857, Durham, NC 27710, USA

Full list of author information is available at the end of the article
}

(c) The Author(s). 2018 Open Access This article is distributed under the terms of the Creative Commons Attribution 4.0 International License (http://creativecommons.org/licenses/by/4.0/), which permits unrestricted use, distribution, and reproduction in any medium, provided you give appropriate credit to the original author(s) and the source, provide a link to the Creative Commons license, and indicate if changes were made. The Creative Commons Public Domain Dedication waiver (http://creativecommons.org/publicdomain/zero/1.0/) applies to the data made available in this article, unless otherwise stated. 


\section{Background}

Undiagnosed diseases are defined as constellations of findings that remain refractory to medical diagnostic approaches. Undiagnosed diseases affect approximately 30 million Americans and include (a) rare diseases that are difficult to identify, (b) atypical presentations of known disorders, and (c) yet to be described diseases [1]. Undiagnosed diseases typically manifest with objective findings, which are clinically measurable on physical examination or through medical testing and these provide tangible targets for further diagnostic approaches (e.g. dysmorphic facies, abnormal biochemical profiles, physical exam demonstrating weakness or abnormal gait). Approximately $80 \%$ of rare and undiagnosed disorders have a genetic basis [1].

The National Institutes of Health established a multi-site network of clinical sites and core laboratories, the Undiagnosed Diseases Network (UDN), in 2013 [2, 3] (https://undiagnosed.hms.harvard.edu) to facilitate the diagnosis/research of undiagnosed and rare diseases. Network-wide, the UDN receives approximately 70 applications each month from adults and parents of children with unexplained illnesses. Application to the UDN is open to all individuals who complete the application with a referral letter from a healthcare provider. The clinical sites make decisions after a comprehensive review of an applicant's medical records. The network accepts about half (51.8\%) for further evaluation. UDN applicants have a wide range of objective and subjective (non-objective) symptoms; however we noticed that many applicants presented primarily or exclusively with subjective findings.

Subjective findings are non-objective, patient-reported symptoms that are not verifiable by physical exam or medical tests [4-6] (e.g. pain, fatigue, weakness that is not substantiated by physical exam). Patients with subjective findings may fall under the diagnostic terms of functional disorder, central sensitivity syndrome, subjective health complaints or even carry the DSM5 diagnosis of somatic symptom disorder. It is difficult to estimate the number of patients affected by these disorders, although it represents a common conundrum in adult primary care medicine [7-9]. Many patients with primarily/ exclusively subjective findings may consider themselves to have undiagnosed diseases and while a small number may have underlying organic disease, the majority are not believed to do so $[6,10]$.

Both patients with objective and subjective findings accrue substantial healthcare costs and seek opinions from multiple specialists, often undergoing extensive/invasive diagnostic testing in their search for a diagnosis [11-14]. Both groups also express chaos in their lives, frustration with negative laboratory tests/procedures, and seek validation of their symptoms [15]. This results in significant personal psychosocial and financial distress and a societal economic impact in both entities [16-19].
However, the likely underlying causes for each group are different: Subjective findings have a number of predisposing risk factors and environmental triggers $[20,21]$ and genetic factors are expected to play at most a modest role [22]. Conversely, approximately $80 \%$ of undiagnosed disorders with clear objective findings have a genetic etiology $[1,17,23]$. Thus, the diagnostic and management approaches for each group differ, with strategies focused on symptom management for the subjective group and further diagnostic pathways, including genomic sequencing for undiagnosed diseases with objective findings.

Utilizing the UDN applicant cohort, we characterize symptoms, referral patterns, and demographic factors among applicants that are and are not accepted for evaluation in the UDN. We sought to provide information to healthcare providers and patients regarding applicants that are suitable for further diagnostic avenues such as the UDN, but more importantly we hope to contribute to a discussion on the need for diagnostic/management/research pathways for applicants who suffer primarily/exclusively from subjective findings.

\section{Methods}

We retrospectively reviewed 201 UDN applications: 151 consecutive applications that were not accepted (Not Accepted Applicants) and 50 randomly selected applications that were accepted (Accepted Applicants) across the seven UDN clinical sites (Additional file 1: Table S1). All applicants provided electronic informed consent as approved by the National Human Genome Research Institute Institutional Review Board under research protocol 15-HG-0130.

Data were provided by the UDN Coordinating Center from the online portal wherein network-wide information is stored. Applications were included if the application decision was issued between $3 / 1 / 2016$ and $1 / 3 / 2017$. We excluded applications that were still under review by the clinical sites and those that lacked a referral letter from a healthcare provider. For all applications, we recorded application outcome, applicant demographics, and application metrics (e.g. referring healthcare provider data). From study referral letters we recorded the number and type of specialty consultations (if any) that were specifically mentioned and medical information described by healthcare providers. For Not Accepted Applicants only, we recorded reasons for non-acceptance and recommendations from the clinical site to the applicant/referring healthcare provider (Additional file 1: Table S2).

On an initial review of 30 Not Accepted Applications, we identified subjective symptoms that were commonly mentioned in referral letters (Additional file 1: Table S3). These were modified through consensus discussions by three authors (NMW, LDMP, VS) and then extracted 
from all the letters. Objective findings varied greatly and were tallied according to their presence in pertinent organ systems (Additional file 1: Table S4). Findings were considered to be objective if the healthcare provider mentioned an abnormal test or examination finding by name. For example, muscle weakness counted as an objective finding only if the provider documented that the weakness was present on physical exam. If specific diagnoses, (e.g. fibromyalgia) were mentioned, these were recorded separately.

Referring healthcare providers included physicians and advance practice providers (e.g. physician assistants and nurse practitioners) who wrote the UDN referral letter. Physicians were classified as primary care physicians or specific specialty when they identified themselves as such, provided letterhead that included this information, or were found via web search to practice family medicine or general internal medicine. Advance practice providers were included in the category "Other" irrespective of their specialty.

Statistical analyses were performed using SPSS 24.0. We used descriptive analyses, student's T-test, Fisher's exact test (FET), ANOVA, and chi-square analyses to analyze factors that affected application outcome. Post-hoc analyses were performed when indicated to infer the significance and direction of individual variables. Binary logistic regression identified associations between the dependent variable of application outcome and key demographic and medical factors.

\section{Results}

Demographics

All UDN clinical sites were represented for the Not Accepted and Accepted applications (Additional file 1: Table S1). There were no significant differences between the Accepted and Not Accepted applicants for gender, race or ethnicity. The Accepted cohort was significantly younger at the time of application and had earlier onset of illness. The duration of illness in both groups was similar but the proportion of an applicant's life being ill was significantly higher for the Accepted applicants. The Not Accepted applications required a significantly longer amount of time for application and medical record review (Table 1).

\section{Healthcare provider referral patterns}

The most common source of referrals to the UDN were primary care physicians (PCP) $(88 / 201,43.7 \%)$. PCP referrals accounted for significantly more applications that were ultimately not accepted [55.6\% of Not Accepted applicants, $8.0 \%$ of Accepted Applicants, FET $p<0.001$ (Table 2, Fig. 1a)]. Accepted Applicants were referred most often by geneticists or neurologists (66\%, Table 2). We found no significant group differences in the number

Table 1 Demographic data of Not Accepted applicants compared to Accepted applicants

\begin{tabular}{|c|c|c|c|}
\hline Feature & Not Accepted $(n=151)$ & Accepted $(n=50)$ & Statistics \\
\hline Age at Application (years, mean \pm SD) & $39.02 \pm 19.11$ & $21.03 \pm 18.83$ & $\begin{array}{l}t=5.78^{* *} \\
\text { Cohen's } d=0.94\end{array}$ \\
\hline Age at Symptom Onset (years, mean \pm SD) & $30.31 \pm 18.75$ & $11.11 \pm 18.0$ & $\begin{array}{l}t=6.3^{* *} \\
\text { Cohen's } d=1.0\end{array}$ \\
\hline Duration of Illness (years, mean \pm SD) & $8.71 \pm 9.91$ & $9.92 \pm 10.06$ & $\begin{array}{l}t=-0.75 \\
\text { Cohen's } d=-0.12\end{array}$ \\
\hline Proportion of Lifetime Being III (percentage) & $28.31 \pm 29.79$ & $66.69 \pm 37.08$ & $\begin{array}{l}t=-6.6^{* *} \\
\text { Cohen's } d=-1.14\end{array}$ \\
\hline Length of Application Review (months, mean \pm SD) & $3.4 \pm 1.73$ & $2.78 \pm 1.76$ & $\begin{array}{l}t=2.17^{*} \\
\text { Cohen's } d=0.35\end{array}$ \\
\hline \multicolumn{4}{|l|}{ Gender } \\
\hline Female & $79(52 \%)$ & $26(52 \%)$ & \multirow[t]{2}{*}{$\mathrm{FET}=1.0$} \\
\hline Male & $72(48 \%)$ & $24(48 \%)$ & \\
\hline \multicolumn{4}{|l|}{ Race } \\
\hline White & $128(84.7 \%)$ & $42(84 \%)$ & \multirow[t]{3}{*}{$x^{2}=0.10$} \\
\hline African-American & $7(4.6 \%)$ & $2(4 \%)$ & \\
\hline Other & $16(10.7 \%)$ & $6(12 \%)$ & \\
\hline \multicolumn{4}{|l|}{ Ethnicity } \\
\hline Hispanic & $11(7.3 \%)$ & $5(10 \%)$ & \multirow[t]{3}{*}{$x^{2}=0.38$} \\
\hline Non-Hispanic & $124(82.1 \%)$ & 40 (80\%) & \\
\hline Not reported & $16(10.6 \%)$ & $5(10 \%)$ & \\
\hline
\end{tabular}


Table 2 Applicant referral sources for all applicants and Reasons for Non-Acceptance for the Not Accepted applications

\begin{tabular}{llll}
\hline Healthcare Provider & Not Accepted & Accepted & Statistics \\
\hline Primary Care Physicians compared to other Specialty Healthcare Providers & $84(55.6 \%)^{\mathrm{a}}$ & $4(8.00 \%)^{\mathrm{a}}$ & $X^{2}=55.48, p<0.001$ \\
Primary Care Physician & $18(11.9 \%)$ & $14(28.0 \%)^{\mathrm{a}}$ & $19(38.0 \%)^{\mathrm{a}}$ \\
Specialist: Neurologist & $8(5.3 \%)^{\mathrm{a}}$ & $13(26 \%)$ \\
Specialist: Geneticist & $41(27.2 \%)$ & & \\
Other (e.g. Allergy Immunologist, Rheumatologist) & & $4(8 \%)$ & FET $p<0.001$ \\
Primary Care Physicians compared to all Other Healthcare Providers & $84(55.6 \%)$ & $46(92 \%)$ & \\
Primary Care Physicians & $67(44.3 \%)$ & & \\
Others & &
\end{tabular}

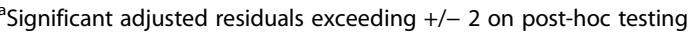

FET Fisher's exact test

of specialist consultations noted by study referral letters (Accepted: $2.62 \pm 2.66$, Not Accepted: $3.12 \pm 2.67, t=-1.14$, $p=0.252$, Cohen's $d$ effect size $=0.18$ ).

\section{Subjective symptoms and objective findings}

The mean number of subjective symptoms was higher in the Not Accepted applicants $(4.1 \pm 3.37)$ relative to the Accepted $(1.7 \pm 2.10, t=4.7, p<0.001$, Cohen's $d$ effect size $=0.85)$. This remains true for subjective symptoms when Accepted applicants are stratified according to whether they did $(N=16)$ or did not $(N=34)$ receive a diagnosis in the UDN (Not Accepted: mean 4.1 \pm 3.37 , Accepted-Not Diagnosed mean 2.06 \pm 2.33 , Accepted-Diagnosed mean $0.94 \pm 1.39$ (ANOVA $F=11.9, p<0.001)$ ). The four most common subjective symptoms (pain, fatigue, headache and memory problems (Additional file 1: Table S3)) were present significantly less frequently in applicants in the Accepted cohort $\left(X^{2}=15.43, p<0.01\right)$ (Fig. 1b). The majority $(114 / 151,75.5 \%)$ of the Not Accepted applicants had at least one objective clinical finding but the mean number of objective findings in the Not Accepted group (1.67 \pm 1.6$)$ was significantly lower than in the Accepted group (3.12 $\pm 1.53, t=-5.4, p<0.001$, Cohen's $d=-0.92)$. This finding remained true when Accepted applicants are again stratified by diagnosis (Not Accepted: mean $1.67 \pm 1.64$, Accepted-Not Diagnosed mean $3.21 \pm 1.72$, Accepted-Diagnosed mean 2.94 \pm 1.24 (ANOVA F $=15.0$, $p<0.001)$ ). In the entire cohort of 201 applicants, there were significantly more subjective symptoms in applicants referred by a primary care physician $(n=88,5.05 \pm 3.50)$ than other healthcare providers $(n=113,2.20 \pm 2.50$, $t=6.2, p<0.001$, Cohen's $d=0.93$ ) (Fig. 1c) and significantly fewer objective findings in those referred by a primary care physician $(1.48 \pm 1.61)$ than other healthcare providers $(2.46 \pm 1.71, t=-4.15, p<0.001$, Cohen's $d=0.6$ ) (Fig. 1c).

\section{Prior diagnoses}

Specific diagnoses were mentioned in some Not Accepted referral letters and the top four: Fibromyalgia, Chronic
Fatigue Syndrome, Mitochondrial disorder and Lyme disease were collectively reported in 28/151 (18.5\%) Not Accepted applications, compared to 6/50 Accepted applications $(12 \%)$ (FET $p>0.05)$. However, the individual diagnosis of fibromyalgia was reported significantly more often in the Not Accepted applications (16/151, 10.6\%) relative to the Accepted $(1 / 50,2 \%$, FET $p=<0.05)$. For the other three diagnoses, there were no significant group differences (FET $p=>0.05$ ), with a frequency of $0-9 \%$ in both groups (Additional file 1: Figure S1).

Reasons for non-acceptance Not Accepted applicants were given reasons for this decision, the most common being that the UDN was unlikely to make a diagnosis, likely reflective of the lack of objective findings in referral letters $(77 / 151,51.0 \%)$. Specific recommendations were provided to $33.8 \%$ (51/151) of the Not Accepted applicants, including recommendations to pursue specific diagnostic tests and seek subspecialty care.

Categorization of illness Neurology was the most common system category selected by both Accepted (24/50, 48\%) and Not Accepted applicants (44/151, 29.1\%, FET $p<0.05)$, followed by Allergy-Immunology $(8 \%$ in Accepted and $10.5 \%$ in Not Accepted) and Musculoskeletal (12\% in Accepted and $7.9 \%$ in Not Accepted). No applicant in either group chose Psychiatry as their disease category; objective psychiatric findings were documented in three Not Accepted healthcare provider letters (Additional file 1: Table S4).

Outcome associations On binary logistic regression with the referring healthcare provider specialty, age at application and age at symptom onset as independent variables and application outcome as the dependent variable, a primary care referral was significantly associated with non-acceptance. Age at application and age at symptom onset were not significantly associated with acceptance status. Similarly, binary logistic regression with the presenting findings showed that higher number 


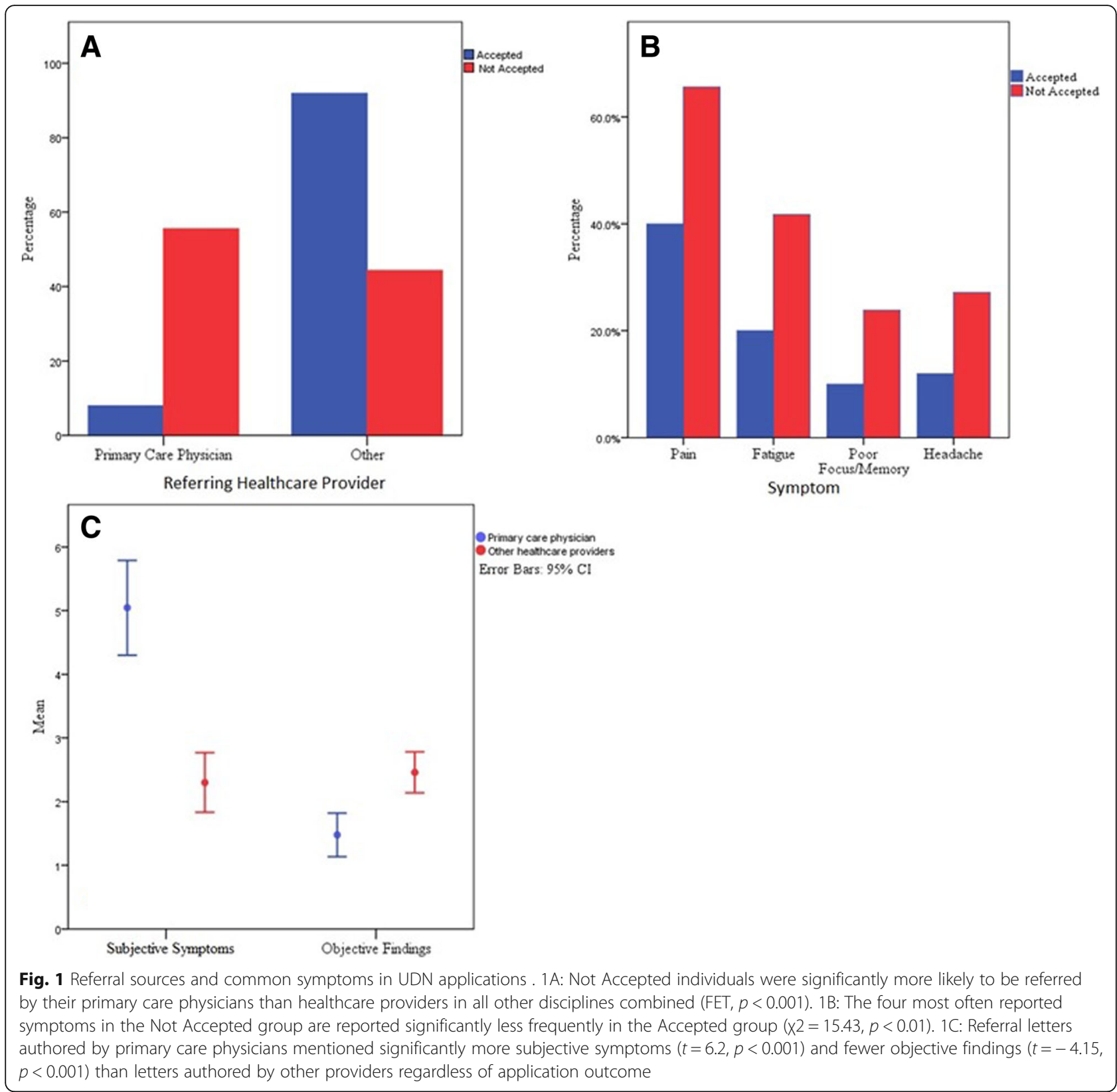

of objective symptoms and fewer subjective symptoms were significantly associated with being accepted (Table 3 ).

\section{Discussion}

Our findings indicate that many applicants that are not accepted to the UDN suffer primarily from subjective symptoms. Further, Not Accepted Applicants were more often adults who had experienced illness for a shorter proportion of their lives and had more non-neurological presentations. Although objective and subjective symptoms were seen in both groups, higher numbers of subjective symptoms were reported for Not Accepted Applicants relative to Accepted subjects. This is true regardless of whether an Accepted applicant was diagnosed by the UDN or not, indicating that a functional disorder was less likely in these remaining still-undiagnosed Accepted applicants, distinguishing them from the Not Accepted applicants. When objective findings were noted in the Not Accepted applicants, these were often unrelated to the reasons for applying to the UDN (e.g. iron deficiency anemia, abnormal thyroid profile) or were non-specific (e.g. positive anti-nuclear antibody). Taken together, we infer that Not Accepted subjects suffer primarily from subjective symptoms that are unlikely to be caused by or related to any objective findings. As such, these applicants are not candidates for further diagnostic 
Table 3 Logistic regression demonstrating relation between key demographic and medical variables and Acceptance or NonAcceptance into the UDN

\begin{tabular}{lll}
\hline Criterion & Odds ratio & $\begin{array}{l}95 \% \text { Confidence } \\
\text { Interval }\end{array}$ \\
\hline $\begin{array}{l}\text { Demographics } \\
\text { Age at UDN Application }\end{array}$ & 1.01 & $0.98-1.05$ \\
Age at onset of symptoms & 1.03 & $0.99-1.07$ \\
$\quad \begin{array}{l}\text { Referring healthcare provider } \\
\text { (Primary care or Other) }\end{array}$ & $8.86^{* * *}$ & $2.84-26.5$ \\
$\begin{array}{l}\text { Medical Findings } \\
\text { Subjective Symptoms }\end{array}$ & $1.40^{* * *}$ & \\
$\quad$ Objective Findings & $0.62^{* * *}$ & $0.19-1.66$ \\
${ }^{*} p<0.05,{ }^{* *} p<0.01,{ }^{* * *} p<0.001$ & &
\end{tabular}

efforts such as those offered by the UDN. Interestingly, the Not Accepted Applications took longer to review, perhaps due to a higher volume of medical records that needed to be reviewed to determine if there were features (such as objective findings beyond subjective symptoms) that would warrant acceptance, although we specifically did not ascertain the reasons for review time.

We also found that primary care physicians referred the largest proportion of applicants to the UDN, but that their referrals had fewer objective findings and were less likely to be accepted. We did not identify a clear reason for this finding, but given that there is no difference in the number of specialty consultations between Not Accepted Applicants and Accepted Applicants, a lack of access to specialists is unlikely. Instead, we presume this indicates that patients with non-objective symptoms continue to seek diagnostic efforts and additional referrals through their PCPs after consultations with specialists do not provide a diagnosis [24].

In the context of the UDN, and perhaps more importantly in the setting of clinical practice, it is important to make a distinction between undiagnosed patients with and without objective findings. Undiagnosed patients with clear objective findings are expected to have a genetic basis in most instances; therefore these diseases can reasonably be expected to be solved (eventually) with additional tests, procedures, and genomic investigations. The UDN is one of several avenues available to pursue a unifying diagnosis. For patients with primarily/exclusively subjective symptoms, additional clinical or genomic investigations are likely to provide a concrete diagnosis in only a small minority $(<5 \%)$ of cases $[10,25]$. There is evidence that risk factors may play a part, such as stress, underlying mood disorders and viral infections, and there are several diagnostic terms, including "functional disorder," "central sensitivity syndrome," or "somatic symptom disorder" that may fit these individuals $[20,21]$. Whether these diagnoses have been discussed with these UDN applicants is not ascertainable from the data available, but it is certain that these applicants attribute their symptoms to a physical illness that requires additional diagnostic efforts.

Despite the dichotomy between undiagnosed patients with and without objective symptoms, the societal and personal impact is remarkably similar. Both groups incur high medical costs and the affected individuals are often significantly disabled. In our recent analysis of UDN narratives, all applicants expressed similar levels of chaos in their lives and frustration with negative laboratory tests/ procedures and many stated that the UDN represented their last hopes for a diagnosis [15].

Although the UDN provides recommendations for applicants and their physicians whenever appropriate, in many cases the onus of continuing medical investigation and treatment falls to primary care physicians, who themselves have limited resources, especially limited time, and few research avenues available to them in their efforts to diagnose/treat patients with subjective findings. There are some research studies that may be an option (https:// mecfs.ctss.nih.gov; https://painconsortium.nih.gov), but there are only a few specific clinical programs (http:// www.chop.edu/centers-programs/center-amplified-muscu loskeletal-pain-syndrome; http://www.amazingkids.org/ Medical-Services/pain-rehabilitation) to address non-objective symptoms, and they are treat primarily children. The diagnostic terms "functional disorder," "central sensitivity syndrome," or "somatic symptom disorder" were almost never identified in referral letters, indicating that PCPs may not be discussing these. Clearly more avenues for management of subjective symptoms are needed beyond the care that PCPs may be able to provide these patients.

This study has some limitations that future research efforts may address. We were unable to ascertain a comprehensive list of subjective/objective findings directly from medical records review. We may have under-ascertained primary care provider referrals because all mid-level providers were classified as "other" regardless of specialty. We were unable to speak with referring providers directly, and future studies that consider these discussions may provide additional insights into a physician's motivations for referral. We were not able to follow applicants after a decision was made and so do not know if any of the Not Accepted applicants received a diagnosis. We also did not have socioeconomic status data available and these may have influenced the types of applications received.

\section{Conclusions}

In conclusion, making a distinction between undiagnosed diseases with and without objective findings enable the affected individuals and their providers, who very often are PCPs, to identify the correct path forward for further management. Confusing subjective symptoms 
for undiagnosed diseases can lead to significant loss of time and resources to identify diagnoses, when further management of the symptoms would be more optimal. Given the prevalence of subjective findings in the general population additional research is warranted in this area to provide clinically appropriate diagnostic guidelines, establish evidence-based treatment options and facilitate honest and effective communication about these illnesses with the patients.

\section{Additional file}

Additional file 1: Figure S1. Referral sources and common symptoms in UDN applications. 1A: Not Accepted individuals were significantly more likely to be referred by their primary care physicians than healthcare providers in all other disciplines combined (FET, $p<0.001$ ). 1B: The four most often reported symptoms in the Not Accepted group are reported significantly less frequently in the Accepted group ( $x 2=15.43, p$ $<0.01)$. 1C: Referral letters authored by primary care physicians mentioned significantly more subjective symptoms $(t=6.2, p<0.001)$ and fewer objective findings $(t=-4.15, p<0.001)$ than letters authored by other providers regardless of application outcome. These materials contain 4 additional data tables and one supplementary figure. (DOCX $63 \mathrm{~kb}$ )

\section{Abbreviations}

PCP: Primary Care Physician; FET, Fisher's Exact Test; UDN: Undiagnosed Diseases Network

\section{Acknowledgements}

We would like to acknowledge all members of the Undiagnosed Diseases Network: David R. Adams, Mercedes E. Alejandro, Patrick Allard, Euan A. Ashley, Mahshid S. Azamian, Carlos A. Bacino, Ashok Balasubramanyam, Hayk Barseghyan, Gabriel F. Batzli, Alan H. Beggs, Hugo J. Bellen, Jonathan A. Bernstein, Anna Bican, David P. Bick, Camille L. Birch, Devon Bonner, Braden E. Boone, Bret L. Bostwick, Lauren C. Briere, Donna M. Brown, Matthew Brush, Elizabeth A. Burke, Lindsay C. Burrage, Shan Chen, Gary D. Clark, Terra R. Coakley, Joy D. Cogan, Cynthia M. Cooper, Heidi Cope, William J. Craigen, Precilla D'Souza, Mariska Davids, Jean M. Davidson, Jyoti G. Dayal, Esteban C. Dell'Angelica, Shweta U. Dhar, Ani Dillon, Katrina M. Dipple, Laurel A. Donnell-Fink, Naghmeh Dorrani, Daniel C. Dorset, Emilie D. Douine, David D. Draper, Annika M. Dries, David J. Eckstein, Lisa T. Emrick, Christine M. Eng, Gregory M. Enns, Ascia Eskin, Cecilia Esteves, Tyra Estwick, Liliana Fernandez, Paul G. Fisher, Brent L. Fogel, Noah D. Friedman, William A. Gahl, Emily Glanton, Rena A. Godfrey, David B. Goldstein, Sarah E. Gould, Jean-Philippe F. Gourdine, Catherine A. Groden, Andrea L. Gropman, Melissa Haendel, Rizwan Hamid, Neil A. Hanchard, Lori H. Handley, Matthew R. Herzog, Ingrid A. Holm, Jason Hom, Ellen M. Howerton, Yong Huang, Howard J. Jacob, Mahim Jain, Yong-hui Jiang, Jean M. Johnston, Angela L. Jones, David M. Koeller, Isaac S. Kohane, Jennefer N. Kohler, Donna M. Krasnewich, Elizabeth L. Krieg, Joel B. Krier, Jennifer E. Kyle, Seema R. Lalani, C. Christopher Lau, Jozef Lazar, Brendan H. Lee, Hane Lee, Shawn E. Levy, Richard A. Lewis, Sharyn A. Lincoln, Allen Lipson, Sandra K. Loo, Joseph Loscalzo, Richard L. Maas, Ellen F. Macnamara, Calum A. MacRae, Valerie V. Maduro, Marta M. Majcherska, May Christine V. Malicdan, Laura A. Mamounas, Teri A. Manolio, Thomas C. Markello, Ronit Marom, Julian A. Martínez-Agosto, Shruti Marwaha, Thomas May, Allyn McConkie-Rosell, Colleen E. McCormack, Alexa T. McCray, Jason D. Merker, Thomas O. Metz, Matthew Might, Paolo M. Moretti, John J. Mulvihill, Jennifer L. Murphy, Donna M. Muzny, Michele E. Nehrebecky, Stan F. Nelson, J. Scott Newberry, John H. Newman, Sarah K. Nicholas, Donna Novacic, Jordan S. Orange, J. Carl Pallais, Christina GS. Palmer, Jeanette C. Papp, Neil H. Parker, Loren DM. Pena, John A. Phillips III, Jennifer E. Posey, John H. Postlethwait, Lorraine Potocki, Barbara N. Pusey, Chloe M. Reuter, Amy K. Robertson, Lance H. Rodan, Jill A. Rosenfeld, Jacinda B. Sampson, Susan L. Samson, Kelly Schoch, Molly C. Schroeder, Daryl A. Scott, Prashant Sharma, Vandana Shashi, Edwin K. Silverman, Janet S. Sinsheimer, Kevin S. Smith, Ariane G. Soldatos, Rebecca C. Spillmann, Kimberly LeBlanc, Joan M. Stoler, Nicholas Stong, Jennifer A. Sullivan, David A. Sweetser, Cynthia J. Tifft, Camilo
Toro, Alyssa A. Tran, Tiina K. Urv, Zaheer M. Valivullah, Eric Vilain, Tiphanie P. Vogel, Daryl M. Waggott, Colleen E. Wahl, Nicole M. Walley, Chris A. Walsh, Michael F. Wangler, Patricia A. Ward, Katrina M. Waters, Bobbie-Jo M. WebbRobertson, Monte Westerfield, Matthew T. Wheeler, Anastasia L. Wise, Lynne A. Wolfe, Elizabeth A. Worthey, Shinya Yamamoto, Yaping Yang, Guoyun Yu, Diane B. Zastrow, Chunli Zhao, Allison Zheng.

\section{Funding}

Research reported in this manuscript was supported by the NIH Common Fund, through the Office of Strategic Coordination/Office of the NIH Director under Award Numbers U01HG007672 (Shashi V and Goldstein DB), U01HG007943 (Jacob HJ) and U01HG007530 (Kohane IS and McCray AT). The content is solely the responsibility of the authors and does not necessarily represent the official views of the National Institutes of Health.

\section{Availability of data and materials}

The datasets generated and/or analyzed during the current study are not publicly available due patient confidentiality but can be redacted and made available from the corresponding author upon reasonable request.

\section{Authors' contributions}

NMW contributed to study design, data collection, data analysis, manuscript preparation and manuscript revision. LDMP and VS contributed to study design, data analysis, manuscript preparation, and manuscript revision. SRH, ATM, KSp, ALW and the Undiagnosed Diseases Network contributed to study design and manuscript revision. KSt contributed to study design. $\mathrm{HC}$, YHJ, $\mathrm{AMR}, \mathrm{CS}, \mathrm{KSC}, \mathrm{RCS}, \mathrm{PM}$, and CE contributed to manuscript revision. All authors read and approved the final manuscript.

\section{Ethics approval and consent to participate}

All applicants provided electronic informed consent with electronic signature as approved by the National Human Genome Research Institute Institutional Review Board under research protocol 15-HG-0130.

\section{Consent for publication}

Consent for research and publication is included in the electronic informed consent as approved by the National Human Genome Research Institute Institutional Review Board under research protocol 15-HG-0130.

\section{Competing interests}

The named authors of this work report no conflicts of interest. Members of the Undiagnosed Diseases Network report the following conflicts of interest: E.A.A. is an advisor to and stockholder in Personalis Inc. D.P.B. is a founder and chief medical officer of Envision Genomics, medical director of Smith Family Clinic LLC, assistant director of Clinical Services Laboratory LLC, and a scientific advisory board member of Genomics England. C.M.E. is a fulltime faculty member of Baylor College of Medicine and provides services as chief medical officer and chief quality officer to Baylor Genetics Laboratory through a professional services agreement. P.G.F. is an associate editor of the Journal of Pediatrics (Elsevier) and a paid consultant. D.B.G. owns equity in two precision medicine companies, Pairnomix and Clarus/EpiPM. J.E.P. is an employee of the Department of Molecular and Human Genetics at Baylor College of Medicine, which has entered a joint venture with Baylor Genetics Laboratory. J.A.R. is a member of the Department of Molecular and Human Genetics at Baylor College of Medicine, which derives revenue from clinical genetic testing offered by Baylor Genetics. M.S.C. is a laboratory director for the HudsonAlpha Clinical Services Lab LLC, which performs fee-for-service clinical laboratory testing. D.A.S. is a member of the Clinical Advisory Board of Baylor Genetics and works for the Department of Molecular and Human Genetics at Baylor College of Medicine, which derives revenue from genetic analyses offered through Baylor Genetics. E.K.S. received honoraria and consulting fees from Merck, grant support and consulting fees from GlaxoSmithKline, and honoraria and travel support from Novartis. P.A.W. is a contract employee for Baylor Genetics, a clinical laboratory that derives income from whole-exome sequencing and other genetic testing.

\section{Publisher's Note}

Springer Nature remains neutral with regard to jurisdictional claims in published maps and institutional affiliations. 


\section{Author details}

'Division of Medical Genetics, Department of Pediatrics, Duke Health, Box 103857, Durham, NC 27710, USA. ${ }^{2}$ Department of Allied Health, University of North Carolina at Chapel Hill, Chapel Hill, NC, USA. ${ }^{3}$ Ethics and Genomics Program, HudsonAlpha Institute for Biotechnology, Huntsville, AL, USA. ${ }^{4}$ Department of Biomedical Informatics, Harvard Medical School, Boston, MA, USA. ${ }^{5}$ National Human Genome Research Institute, NIH, Bethesda, MD, USA.

Received: 19 December 2017 Accepted: 9 August 2018

Published online: 22 August 2018

\section{References}

1. https://globalgenes.org. Rare Disease Statistics 2015.

2. Gahl WA, Wise AL, Ashley EA. The undiagnosed diseases network of the National Institutes of Health: a National Extension. JAMA. 2015;314(17):1797-8.

3. Ramoni RB, Mulvihill JJ, Adams DR, et al. The undiagnosed diseases network: accelerating discovery about health and disease. Am J Hum Genet. 2017; 100(2):185-92.

4. Nettleton S, Watt I, O'Malley L, Duffey P. Understanding the narratives of people who live with medically unexplained illness. Patient Educ Couns. 2005;56(2):205-10

5. McAndrew LM, Phillips LA, Helmer DA, et al. High healthcare utilization near the onset of medically unexplained symptoms. J Psychosom Res. 2017;98:98-105.

6. Eriksen HR, Ursin H. Subjective health complaints, sensitization, and sustained cognitive activation (stress). J Psychosom Res. 2004;56(4):445-8.

7. Nettleton S. 'I just want permission to be ill': towards a sociology of medically unexplained symptoms. Soc Sci Med. 2006;62(5):1167-78.

8. Haller $\mathrm{H}$, Cramer $\mathrm{H}$, Lauche R, Dobos $\mathrm{G}$. Somatoform disorders and medically unexplained symptoms in primary care. Deutsches Arzteblatt Int. 2015;112(16):279-87.

9. Rasmussen EB. Balancing medical accuracy and diagnostic consequences: diagnosing medically unexplained symptoms in primary care. Sociol Health IIIn. 2017;39(7):1227-41.

10. Crimlisk HL, Bhatia K, Cope H, David A, Marsden CD, Ron MA. Slater revisited: 6 year follow up study of patients with medically unexplained motor symptoms. BMJ. 1998;316(7131):582-6.

11. Konnopka A, Kaufmann C, Konig HH, et al. Association of costs with somatic symptom severity in patients with medically unexplained symptoms. J Psychosom Res. 2013;75(4):370-5.

12. Konnopka A, Schaefert $R$, Heinrich $S$, et al. Economics of medically unexplained symptoms: a systematic review of the literature. Psychother Psychosom. 2012;81(5):265-75.

13. Chaturvedi SK, Desai G. Measurement and assessment of somatic symptoms. Int Rev Psychiatry (Abingdon, England). 2013;25(1):31-40.

14. Burton C, McGorm K, Richardson G, Weller D, Sharpe M. Healthcare costs incurred by patients repeatedly referred to secondary medical care with medically unexplained symptoms: a cost of illness study. J Psychosom Res. 2012;72(3):242-7.

15. Spillmann RC, McConkie-Rosell A, Pena $L$, et al. A window into living with an undiagnosed disease: illness narratives from the undiagnosed diseases network. Orphanet J Rare Dis. 2017;12(1):71.

16. Angelis A, Tordrup D, Kanavos P. Socio-economic burden of rare diseases: a systematic review of cost of illness evidence. Health Policy. 2015;119(7):964-79.

17. Eurordis. Rare Diseases: Understanding this public health priority. wwweurordisorg. 2005

18. Carmichael N, Tsipis J, Windmueller G, Mandel L, Estrella E. "is it going to hurt?": the impact of the diagnostic odyssey on children and their families J Genet Couns. 2015;24(2):325-35.

19. Shashi V, McConkie-Rosell A, Rosell B, et al. The utility of the traditional medical genetics diagnostic evaluation in the context of next-generation sequencing for undiagnosed genetic disorders. Genet Med. 2014;16(2):176-82.

20. Hamilton WT, Gallagher AM, Thomas JM, White PD. Risk markers for both chronic fatique and irritable bowel syndromes: a prospective case-control study in primary care. Psychol Med. 2009;39(11):1913-21.

21. Moss-Morris R, Spence M. To "lump" or to "split" the functional somatic syndromes: can infectious and emotional risk factors differentiate between the onset of chronic fatigue syndrome and irritable bowel syndrome? Psychosom Med. 2006;68(3):463-9.
22. Kato K, Sullivan PF, Evengard B, Pedersen NL. A population-based twin study of functional somatic syndromes. Psychol Med. 2009:39(3):497-505.

23. Chong JX, Buckingham KJ, Jhangiani SN, et al. The genetic basis of Mendelian phenotypes: discoveries, challenges, and Opportunities. Am J Hum Genet. 2015;

24. Hoedeman R, Blankenstein AH, Van der Feltz-Cornelis CM, Krol B, Stewart R, Groothoff JW. Consultation letters for medically unexplained physical symptoms in primary care. Cochrane Database Syst Rev. 2010;(12):Cd006524.

25. Zarovnaya EL, Pallatroni HF, Hug EB, et al. Atypical teratoid/rhabdoid tumor of the spine in an adult: case report and review of the literature. JNeurooncol. 2007;84(1):49.

\section{Ready to submit your research? Choose BMC and benefit from:}

- fast, convenient online submission

- thorough peer review by experienced researchers in your field

- rapid publication on acceptance

- support for research data, including large and complex data types

- gold Open Access which fosters wider collaboration and increased citations

- maximum visibility for your research: over $100 \mathrm{M}$ website views per year

At $\mathrm{BMC}$, research is always in progress.

Learn more biomedcentral.com/submissions 